\title{
Nucleophilic Attack on Nitrogen in Tetrazines by Silyl-Enol Ethers
}

\author{
Simon D. Schnell, Mauro Schilling, Jan Sklyaruk, Anthony Linden, Sandra Luber,*
} and Karl Gademann*

\section{Department of Chemistry \\ University of Zurich}

Winterthurerstrasse 190, 8057 Zurich, Switzerland

E-mail: karl.gademann@chem.uzh.ch; sandra.luber@chem.uzh.ch

* Corresponding Authors

Supporting information for this article is given via a link at the end of the document. 


\section{Abstract}

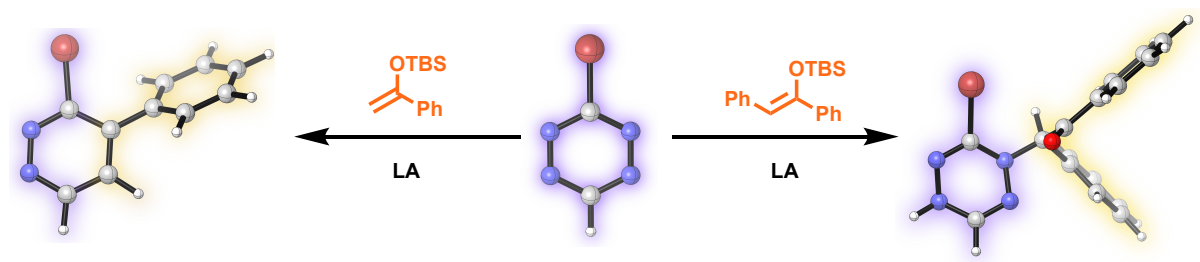

The nucleophilic addition to nitrogen in 3-monosubstituted $s$-tetrazines under mild conditions is reported, by using silyl-enol ethers as the nucleophiles and mediated by $\mathrm{BF}_{3}$. The preference for this azaphilic addition over the usually observed inverse electron demand Diels-Alder reactions was determined experimentally and evaluated theoretically. In this regard, the influence of the effect of $\mathrm{BF}_{3}$-coordination to $s$-tetrazines was investigated thoroughly. The substrate dependency of this unusual reaction was rationalized by determination of the activation barriers and on the basis of the activation strain model by employing density functional theory. Lastly, the decomposition of the unstable adducts was examined and an interesting rearrangement to a triazine derived structure was observed. 


\section{Introduction}

Aromatic compounds have been central to chemistry over the last two decades, and discoveries concerning their properties, ${ }^{[1]}$ their preparation, ${ }^{[2]}$ and their production have impacted society as a whole, from dyes and colorants ${ }^{[3]}$ to drugs ${ }^{[4]}$ and crop protection agents. ${ }^{[5]}$ The chemistry of aromatic compounds fills textbook chapters, ${ }^{[6]}$ and students in many disciplines learn the basic reactivity of these compounds, such as electrophilic or nucleophilic aromatic substitution. ${ }^{[7]}$ The latter reaction classically involves nucleophilic attack on an electron-deficient aromatic system with subsequent elimination of a suitable leaving group (Scheme 1, top left). ${ }^{[8]}$ Nucleophilic attack on aromatic compounds without elimination is generally not favored, as aromaticity is not restored. In addition, students also learn that the $\mathrm{N}$ atom in nitrogen-containing aromatics usually serves as a nucleophile in many different reactions, which results in a chemical bond to an electrophilic partner. ${ }^{[9]}$
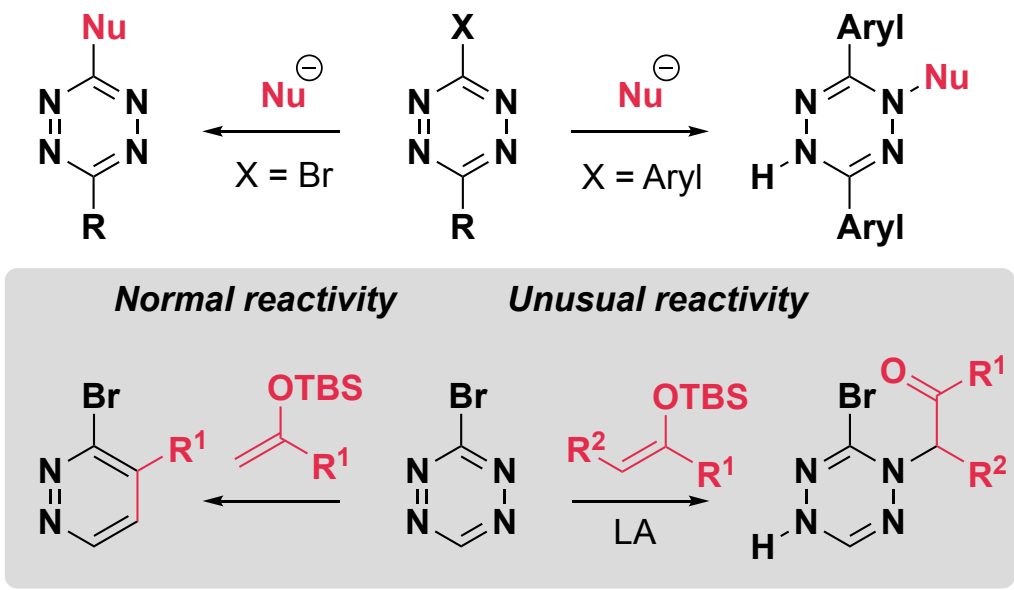

Scheme 1. Reactivity of $s$-tetrazines.

In this communication, and in contrast to all these textbook examples, we report on an unusual nucleophilic attack on the electrophilic nitrogen atom in electron-deficient tetrazines in an Umpolung reaction (Scheme 1, bottom right). In addition, no elimination and no concomitant restoration of aromaticity are observed, as this reaction strikingly leads to the corresponding non-aromatic dihydroderivatives.

$s$-Tetrazines have emerged as the heterocycles of choice for a wide range of applications, in particular with regard to bio-orthogonal chemistry. ${ }^{[10]}$ In such settings, the inverse electron demand [4+2] DielsAlder (iEDDA) reaction of $s$-tetrazines with strained alkenes and alkynes enabled successful conjugation reactions, even at biologically relevant concentrations. ${ }^{[1]}$ As pioneered by Sauer and co-workers, ${ }^{[12]}$ 
olefins typically result in the corresponding aromatic pyridazines, a process that can also be accelerated by Lewis acids. ${ }^{[13]}$

In contrast to cycloaddition reactions, direct addition reactions of nucleophiles to $\mathrm{N}$-containing aromatics have rarely been described in the literature, and are generally limited to 1,2,3-benzotriazoles, 1,2,3triazines, and 1,2,4,5-tetrazines. ${ }^{[14,15]}$ To the best of our knowledge, there are only three reports in the literature on the addition of hard organometallic reagents, such as RLi or RMgX, to the tetrazine core. ${ }^{[14}$, ${ }^{16,17]}$ Neugebauer and Siegel were first to react unsubstituted $s$-tetrazine with methyl magnesium iodide to give 1,4-dihydro-1-methyl-s-tetrazine, ${ }^{[17]}$ which was corroborated independently at the same time by Hunter and Neilson. ${ }^{[16]}$ Subsequently, the role of different metal ions of aryl and alkyl organometallic reagents was then investigated by Kotschy and coworkers for 3,6-disubsituted $s$-tetrazines. ${ }^{[14,18]}$ This reactivity was only observed with strong organometallic nucleophiles and thus cannot generally be applied to 3-monosubstituted s-tetrazines, because the instability of these compounds towards strong bases results in degradation of the tetrazine core. ${ }^{[19]}$ In the context of our research on the chemistry of 3monosubstituted $s$-tetrazines and specifically 3-bromotetrazine (3-Br-Tet) (1), a small $s$-tetrazine building block for the labelling of macromolecules previously reported by our group and others, ${ }^{[20]}$ we investigated their reactivity with silyl-enol ethers. In this context, we show that the reactivity of silyl-enol ethers can switch from an unprecedented nucleophilic (azaphilic) addition of an unsaturated system to the $\mathrm{N}$ atom of $s$-tetrazine by employing bulky reagents to cycloaddition in the presence of less bulky groups, thereby producing pyridazines.

\section{Results and Discussion}

Preliminary experiments revealed that a catalyst was necessary in order to promote the reaction of silylenol ethers with 3-Br-Tet (1). Based on previous reports by Wegner and co-workers, who used a bidentate bisborane Lewis acid to activate $s$-tetrazines, we were interested to see if commercially available $\mathrm{BF}_{3}$. $\mathrm{OEt}_{2}$ could replace the more complex bisborane Lewis acid. (Scheme 2). The reaction of 3-Br-Tet (1) with TBS-silyl-enol ether 2 cleanly produced 3-bromo-4-phenylpyridazine (3) in $82 \%$ yield and the constitution was confirmed by a single crystal X-ray structure analysis. Remarkably, the reaction proved to be very fast (15 min, room temperature) and regioselective (single isomer observed). Interestingly, when the higher substituted silyl-enol ethers $\mathbf{4}$ and $\mathbf{5}$ were employed, the outcome of the reaction changed drastically. When methyl-substituted silyl-enol ether 4 was reacted with 3-Br-Tet (1), the pyridazine 6 was only observed as the minor product of this transformation in low conversion (12\%) together with a major unknown compound, as judged by ${ }^{1} \mathrm{H}-\mathrm{NMR}$ spectroscopy. 

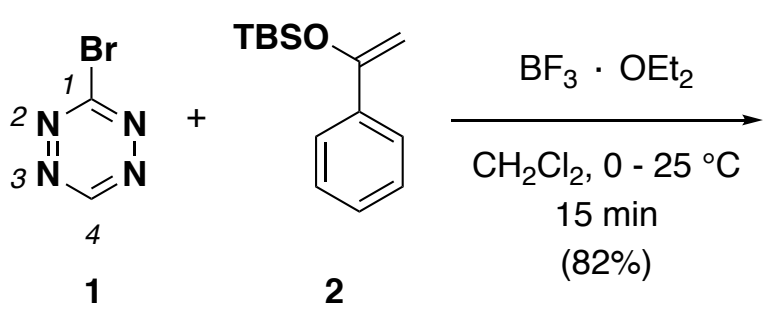

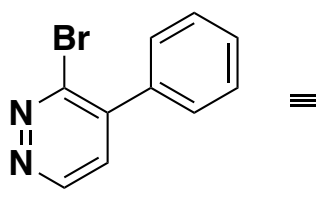

3

(single product)

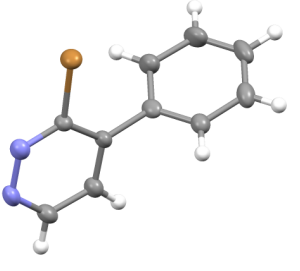

molecular structure

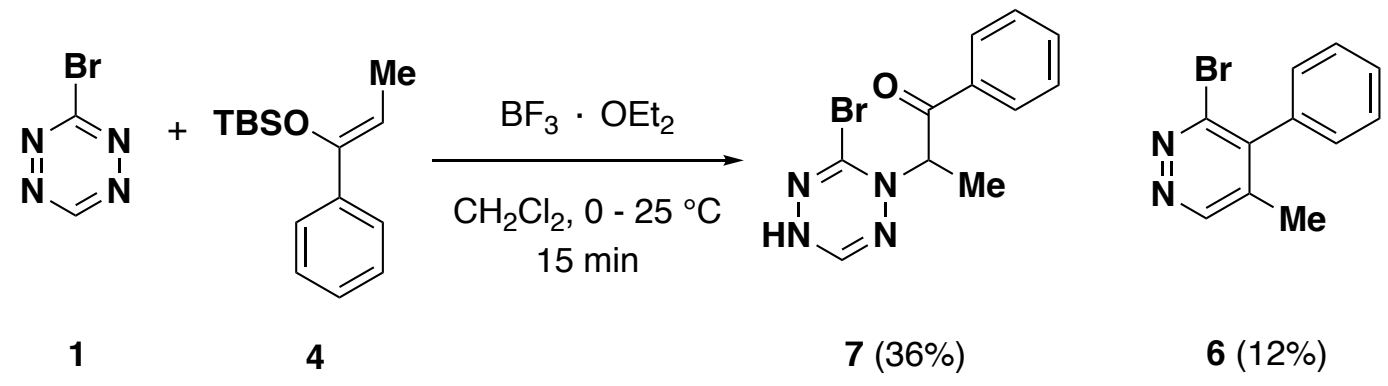

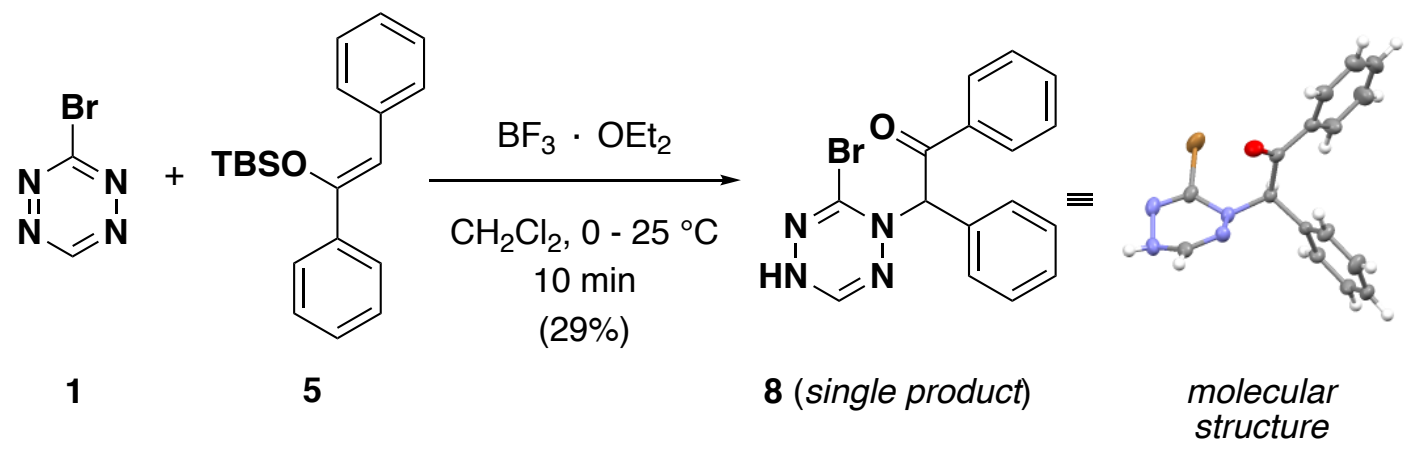

Scheme 2. Reactivity of silyl-enol ethers with 3-Br-Tet (1).

The major product was assigned to be the azaphilic addition product $\mathbf{7}$ by extensive NMR analysis. However, since an inseparable mixture of adduct and pyridazine was obtained, we employed the bulkier silyl-enol ether $\mathbf{5}$ in the hope of achieving exclusive selectivity and easy separation from impurities. In this case, a single product was obtained (as observed by UHPLC-MS and NMR analysis) and singlecrystal X-ray structure analysis unambiguously confirmed the structure of $\mathbf{8}$ to correspond to the azaphilic addition product. In order to gain insight into this unique and novel reactivity of silyl-enol ethers with 3 Br-Tet (1) and to understand the crucial role of the Lewis acid, we extensively studied theoretically the effect of the coordination of $\mathrm{BF}_{3} \cdot \mathrm{OEt}_{2}$ to 3 -Br-Tet $(\mathbf{1})$ and the influence of substituents on the silyl-enol ether. We analyzed the frontier molecular orbitals (FMOs) of the tetrazine by means of condensed Fukui functions (electrophilic: $f^{-}$, nucleophilic: $f^{+}$) (see supporting information for a detailed description of the used computational methods and their validation). Based on our preliminary experimental observations and theoretical calculations on the relative stability of $\mathrm{BF}_{3}$ adducts, we propose the coordination of a single Lewis acid moiety at N-3 of 3-Br-Tet (1) (see Table S8). Further, we found that 
independent of the coordination site of the $\mathrm{BF}_{3}$, there is no alteration of the symmetry of the FMOs (see Figures S1 and S2). However, when comparing the relative local electrophilicity ${ }^{[21]}$ index calculated from condensed Fukui functions by conceptual DFT, ${ }^{[22]}$ we found, that the coordination of $\mathrm{BF}_{3}$ leads to a significant depletion of the electron density at the para nitrogen atom. Bearing this result in mind, this nitrogen atom becomes more susceptible to an azaphilic attack (see Figure 1) and a high regioselectivity for azaphilic attack is expected. In addition to this, upon coordination of the Lewis acid, a steady decrease in the orbital energies of all the frontier orbitals (HOMO-1, HOMO, LUMO, and LUMO+1) can be observed (see Figure S4). In accordance with FMO theory, we observe that the stabilization of the orbitals correlates with an increased reactivity. ${ }^{[23]}$ The decrease in the energies of the orbitals follows the same trend as the relative stability of the $\mathrm{BF}_{3}$ adducts, i.e. interactions between the Lewis acid and the bromosubstituent are avoided.

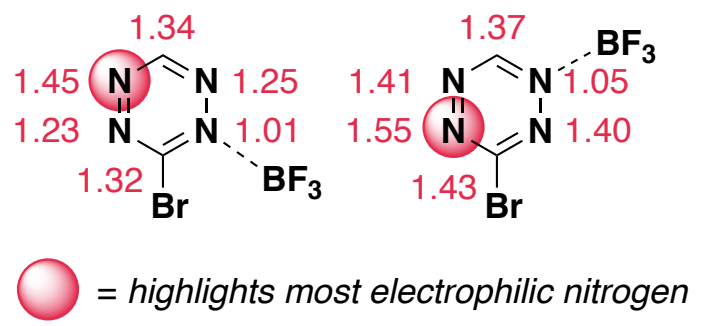

Figure 1. Relative local electrophilicity index of the $\mathrm{BF}_{3}$ adducts relative to native 3-Br-Tet (1). This dimensionless quantity helps to rationalize the reactivity of tetrazine towards an azaphilic addition. The most electrophilic site (highlighted) corresponds to the nitrogen atom that is found to undergo the azaphilic addition reaction.

In order to rationalize the selectivity towards either the azaphilic addition for enol ethers $\mathbf{4}$ and $\mathbf{5}$ or the iEDDA reaction for enol ether $\mathbf{2}$, we employed the active strain model (ASM). ASM was demonstrated to be a suitable tool for decomposing the activation barriers into different components, namely the distortion energy required to deform the reactants and the interaction energy gained through stabilizing interactions. ${ }^{[24]}$ The ability of the dienophiles used in this study to undergo an iEDDA like reaction is clearly dependent on the steric demand imposed by the alkyl/aryl substituent. This can be seen from the distinctive difference in the deformation energy as a function of the $\mathrm{C}-\mathrm{C}$ bond formation, which follows the expected trend $E_{\text {strain }}: \mathrm{Ph}>\mathrm{Me}>\mathrm{H}$ (see Figure 2). On the other hand, the azaphilic addition is, as expected, only marginally affected by the nature of substituents of the dienophile as can be seen from the almost identical $E_{\text {strain }}$ and $E_{\text {int }}$ energies (see Figure S5). 


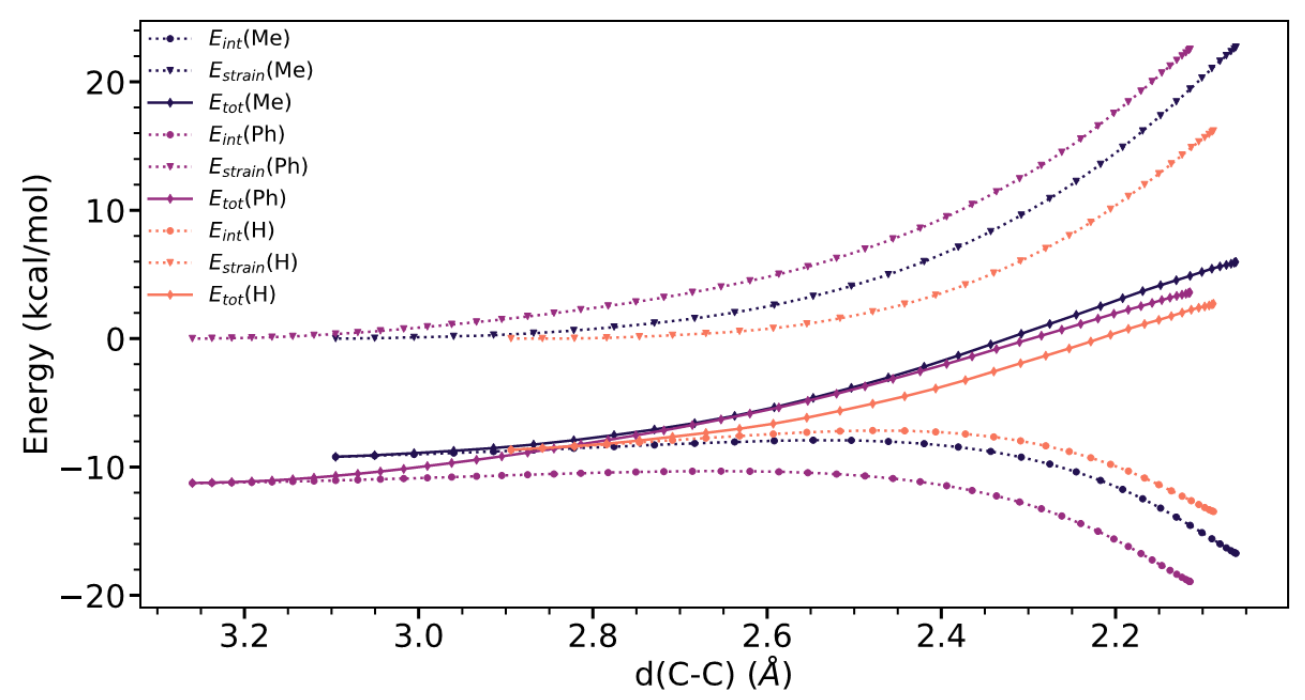

Figure 2. Plot of the total electronic energy $\left(E_{\text {tot }}\right)$, distortion energy $\left(E_{\text {strain }}\right)$, and interaction energy $\left(E_{\text {int }}\right)$ versus the bond distance of the $\mathrm{C}-\mathrm{C}$ bond that is to be formed between the dienophiles ( $\mathrm{H}(2)$, Me (4), and $\mathrm{Ph}(\mathbf{5}))$ and 3-Br-Tet (1) without the aid of a Lewis acid. Note, $\left(E_{\text {strain }}\right)$ of the structures representing the associated reactant (AR) was set to zero for all derivatives.

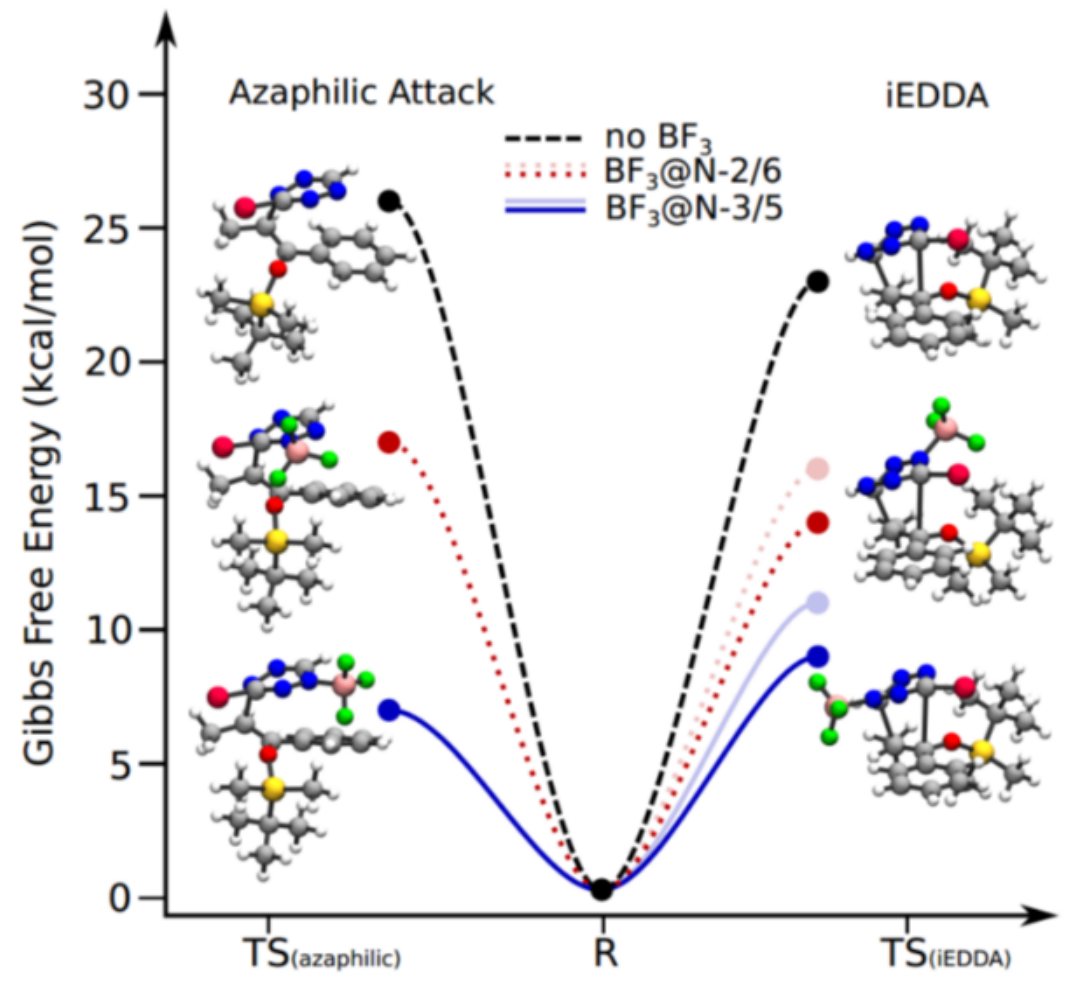

Figure 3. Gibbs free energy profile of the azaphilic attack and Diels-Alder like reaction for the methylderivative 4 with and without $\mathrm{BF}_{3}$ adducts. In the case of the iEDDA reaction, only $\mathrm{BF}_{3} @ \mathrm{~N}-5$ and 
$\mathrm{BF}_{3} @ \mathrm{~N}-2$ are shown. Note that in the case of the azaphilic attack, only the nitrogen atoms in the para or meta position are expected to react.
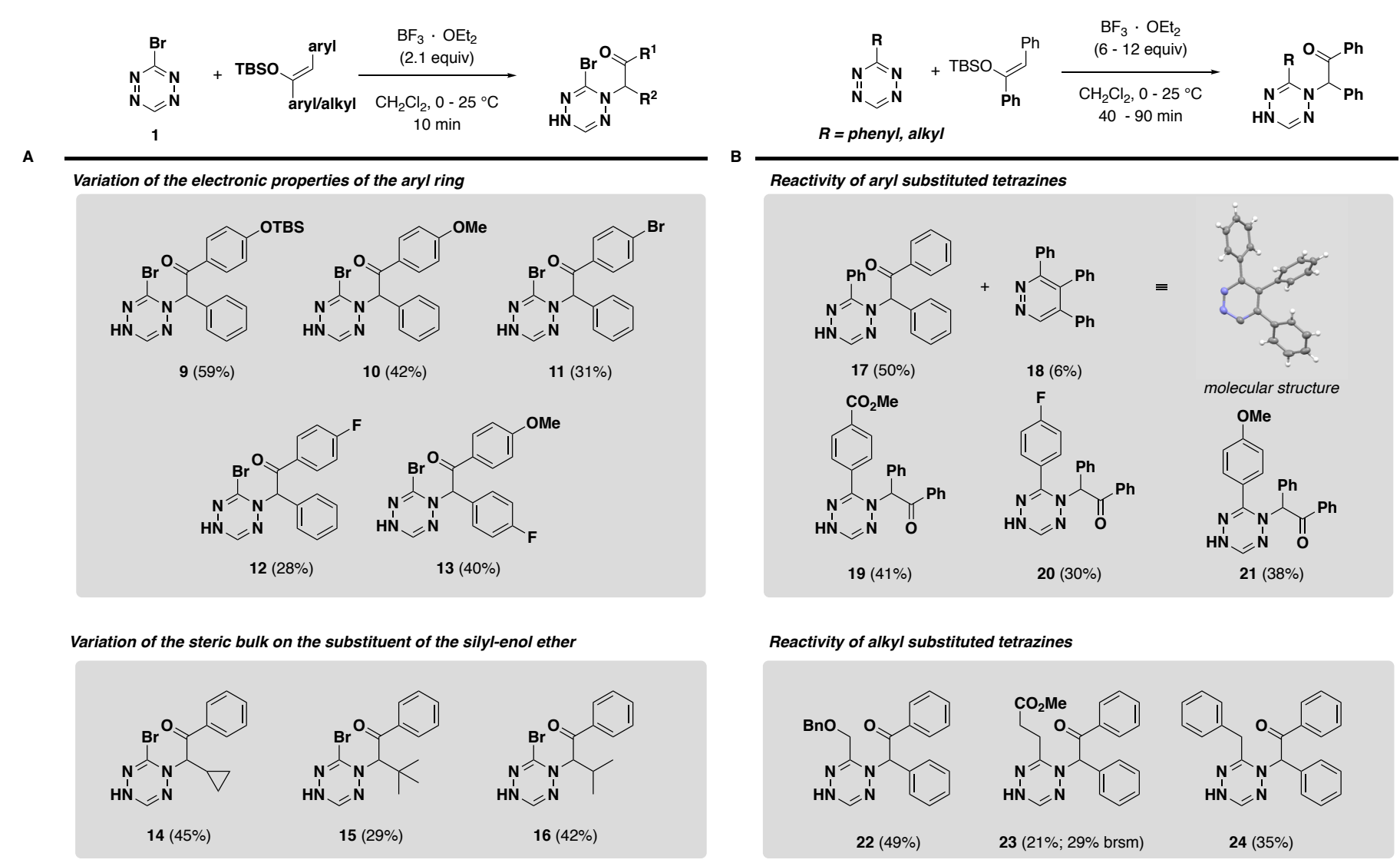

Scheme 3. Azaphilic addition to 3-bromo, 3-aryl, and 3-alkyl substituted $s$-tetrazines.

The conclusions drawn from the ASM analysis are supported by the calculated activation barriers for all three silyl-enol ethers $\mathbf{2 , 4}$ and $\mathbf{5}$ in the presence and absence of $\mathrm{BF}_{3}$, respectively (see Tables S6 and S7). For both iEDDA and azaphilic attack, independent of the nature of the silyl-enol ether, we find the barriers to be the lowest if $\mathrm{BF}_{3}$ is coordinated to the $\mathrm{N}-3$ or $\mathrm{N}-5$ position, as suggested by the local electrophilicity index. The experimentally observed preference for either the azaphilic addition or the iEDDA reaction can be rationalized by the calculated activation energies, which are 21 (nuc.) vs $26 \mathrm{kcal} / \mathrm{mol}$ (iEDDA) in the case of the phenyl derivative, 7 vs. $11 \mathrm{kcal} / \mathrm{mol}$ in the case of the methyl derivative, and 15 vs 12 $\mathrm{kcal} / \mathrm{mol}$ in the case of the non-substituted allyl (see Figure 3 and Tables S6 and S7).

Based on the experimental and theoretical insights observed, we studied the steric and electronic influence of the substituents of the trisubstituted silyl-enol ethers (Scheme 3A). In addition, the effect of aryl and alkyl substitution on the $s$-tetrazine core was probed experimentally (Scheme 3B). As expected, introducing an electron donating group in the para-position of the aromatic ring increased the yield up to 59\% (compounds 9 and 10). In contrast, para-bromo or para-fluoro substituents apparently had no effect 
on the yield of the reaction and led to the isolation of the azaphilic addition products $\mathbf{1 1}$ and $\mathbf{1 2}$ in 31 and $28 \%$ yield, respectively. The introduction of a "push-pull” system slightly increased the yield compared with 8; compound 13 could be isolated in $40 \%$ yield. With these results in hand, we tried several alkyl residues in order to determine the minimum steric requirement for obtaining exclusively the azaphilic addition product, bearing in mind that a methyl substituent afforded a mixture. Interestingly, only for the respective cyclopropyl TBS-silyl enol ether, the pyridazine could be observed in trace amounts in UHPLC-MS. The main product proved to be $\mathbf{1 4}$, which was isolated in $45 \%$. For a tert-butyl or iso-propyl group, the azaphilic addition products 15 and 16 remained the only observed products of this transformation and could be isolated in 29 and $42 \%$ yield, respectively.

Having established the generality of the presented reaction with 3-Br-Tet (1), we were intrigued to see if this reactivity can be transferred directly to more electron rich alkyl and aryl substituted tetrazines (Scheme 3B). Interestingly, a larger excess of $\mathrm{BF}_{3} \cdot \mathrm{OEt}_{2}$ was necessary to achieve synthetically useful conversions and yields. Possible reasons for this requirement could involve stronger Lewis basicity of corresponding reaction products, binding multiple equivalents of $\mathrm{BF}_{3}$. Along these lines, aryl substituted tetrazines required 6 equiv of Lewis acid and alkyl substituted tetrazines required 12 equiv of $\mathrm{BF}_{3} \cdot \mathrm{OEt}_{2}$, together with longer reaction times compared to azaphilic attack on 3-Br-Tet (1). The longer reaction time can be correlated with the higher electron density within the tetrazine ring resulting from the electron donating ability of the alkyl or aryl substituents. This effect of the substituent then leads to a less favorable azaphilic addition pathway. Nevertheless, also for alkyl and aryl substituents, the azaphilic addition pathway is favored over the inverse electron demand Diels-Alder reaction, and only in the case of compound 17, was the pyridazine 18 obtained in isolable amounts as a minor-byproduct (6\%) (Scheme 3B). Electron-acceptors, as well as donors, on the tetrazine core were tolerated under these conditions and led to the formation of dihydrotetrazines $19-21$ in moderate yields. Several alkyl substituted dihydrotetrazines $\mathbf{2 2}-\mathbf{2 4}$ could also be isolated and were obtained in $21-49 \%$ yield and with exclusive regioselectivity. In order to gain further experimental insight into the transformation, explored whether or not a phenyl group at $\mathrm{C} 1$ in the silyl-enol ether is mandatory (Scheme 4), or if a bulky alkyl residue also promotes azaphilic addition. 
<smiles>CC(C)(C)C(=Cc1ccccc1)OC#[SH]</smiles>

1<smiles>COC1=CCCC[C+]1[n+]1ncnnc1Br</smiles>

1<smiles>Brc1nncn[nH+]1</smiles>

1
25

29

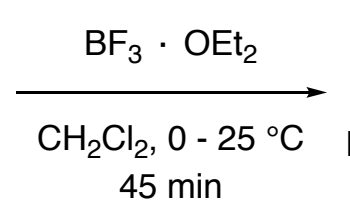

$(42 \%)$<smiles>CC(C)(C)C(=O)C(c1ccccc1)N1N=CNN=C1Br</smiles>

26

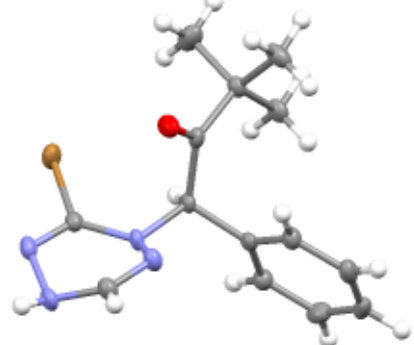

molecular structure

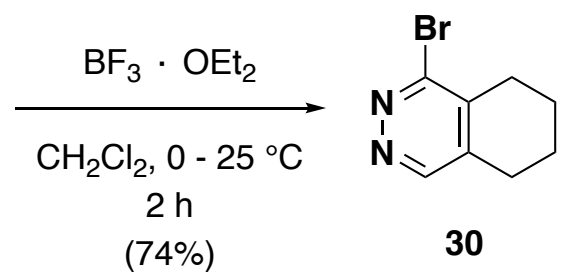

(74\%)<smiles>Brc1nncc(-c2ccccc2)c1-c1ccccc1</smiles>

28

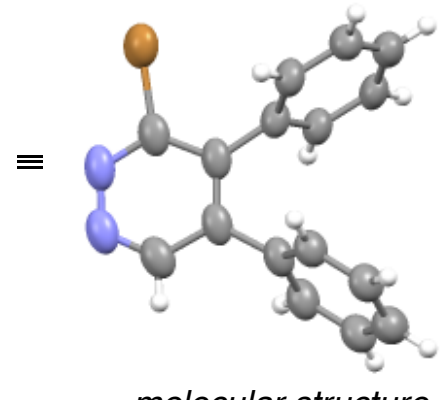

molecular structure

Scheme 4. Reactivity of TES-silyl-enol ethers, enamines and cyclic silyl-enol ethers.

Along these lines, we synthesized TES-silyl-enol ether 25; the corresponding TBS-silyl-enol ether remained synthetically inaccessible despite employing several enolization procedures. The respective TES-silyl-enol ether was subjected to the standard reaction conditions, which led to smooth and exclusive formation of dihydrotetrazine $\mathbf{2 6}$ and the adduct could be isolated in $42 \%$ yield. The structure was unambiguously verified via single-crystal X-ray structure analysis. Additionally, we probed the result of the ASM study experimentally, by correlating the observed azaphilic addition reactivity with the double bond geometry of the silyl-enol ethers being $Z$. In order to evaluate this hypothesis, the cyclic silyl-enol ether 29 (locked in an $E$ configuration) was reacted under the conditions reported herein, and only the bicyclic pyridazine $\mathbf{3 0}$ was obtained; the corresponding azaphilic addition product remained elusive. TMS-enol ethers were used in this case, because higher yields were obtained in comparison with the TBS analogs. This result thus provides experimental evidence for the importance of the double bond geometry in the silyl-enol ethers, if azaphilic addition is the desired reaction pathway.

Additionally, we prepared enamine 27, ${ }^{[25]}$ which features an $(E)$ configuration. Also in this case, pyridazine 28 was the only observable product, being obtained in good yield (75\%). Remarkably, this highly substituted pyridazine was obtained with exclusive selectivity without the use of a Lewis acid in 
short reaction times. Since the synthesis of highly substituted pyridazines with high regiocontrol still remains a challenge, this method potentially gives an easy entry to the synthesis of complex pyridazines bearing a Br-atom for further functionalization via cross-coupling reactions. ${ }^{[26]}$

Lastly, we investigated the decomposition of the adducts using dihydrotetrazine $\mathbf{8}$ as a model substrate. Although all described azaphilic addition products could be isolated and purified via standard silica gel column chromatography, the obtained compounds have limited stability in solution. According to UHPLC-MS, conversion to a single new compound was observed, when stirring dihydrotetrazine 8 in $\mathrm{CH}_{2} \mathrm{Cl}_{2}$ over a period of 3 days. After several attempts, we were able to obtain suitable crystals of the $\mathrm{HBr}$ salt of the rearranged product $\mathbf{3 1}$ for a single-crystal X-ray structure analysis (Scheme 5).

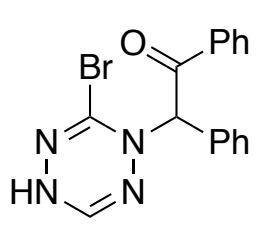

8
$\mathrm{CH}_{2} \mathrm{Cl}_{2}, 3 \mathrm{~d}$

$(67 \%)$

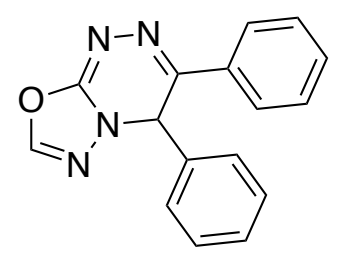

31 $\equiv$
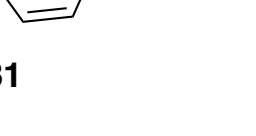

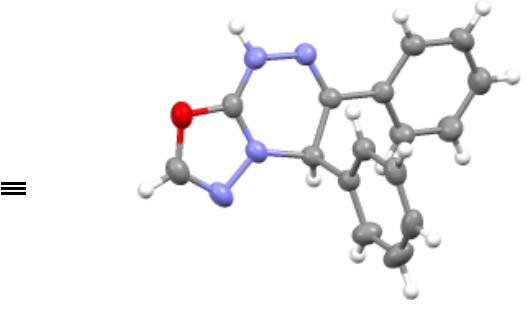

molecular structure the of $\mathrm{HBr}$ salt of $\mathbf{3 1}$

Scheme 5. Rearrangement of dihydrotetrazine $\mathbf{8}$ to triazine derived $\mathbf{3 1}$.

To our surprise, the fused heterocyclic structure $\mathbf{3 1}$ was formed through a novel rearrangement. Derivatives of this triazine scaffold with a different substitution pattern were obtained earlier via a different route; the synthesis of this heterocyclic scaffold from tetrazines is unprecedented, to our knowledge. ${ }^{[27]}$ In contrast to previous syntheses of these heterocycles, where the precursors are 4acylamino-3-oxo-6-aryl-2,3,4,5-tetrahydrotriazines, s-tetrazines give easy access to these heterocyclic scaffolds with a novel substitution pattern. Studies on the mechanism of this unprecedented rearrangement are currently being conducted in our laboratory.

\section{Conclusion}

In conclusion, to the best of our knowledge, this is the first report of an azaphilic addition to $s$-tetrazines induced by the mildly nucleophilic silyl-enol ether reagent. This reactivity opens a completely new and unprecedented reaction pathway in addition to the expected iEDDA for $s$-tetrazines with unsaturated compounds. In contrast, rare previous reports observed azaphilic additions only with strongly nucleophilic organometallic reagents (Grignard reagents, organolithium reagents), ${ }^{[28]}$ whereas we could induce this reactivity using a mild protocol with $\mathrm{BF}_{3}$ as a Lewis acid mediator and a silyl-enol ether as a mild carbon nucleophile. Along these lines, unprecedented dihydrotetrazine scaffolds of the structure of $\mathbf{8}$, were 
obtained. Experimental findings were supported by X-ray crystal-structure analyses, which unambiguously confirmed the structures of the adducts, as well as the exclusive regioselectivity. Theoretical calculations of the reaction could provide additional valuable insights into the formation of the dearomatized dihydrotetrazines. Finally, a novel rearrangement of the dihydrotetrazine core to a triazine $\mathbf{3 1}$ derived bicyclic scaffold was recognized.

\section{Supporting Information}

Detailed experimental procedures and characterization data are included in the Supporting Information of this manuscript together with crystallographic data and the employed computational methods. CCDC2036281-2036286 contain the supplementary crystallographic data for this paper. The data can be obtained free of charge from The Cambridge Crystallographic Data Center via www.ccdc.cam.ac.uk/structures.

\section{Author Information}

\section{Corresponding Authors}

Prof. Dr. Karl Gademann - Department of Chemistry, University of Zurich, Winterthurerstrasse 190, 8057, Zurich. Email: Karl.gademann@chem.uzh.ch Prof. Dr. Sandra Luber - Department of Chemistry, University of Zurich, Winterthurerstrasse 190, 8057, Zurich. Email: $\quad$ sandra.luber@chem.uzh.ch

\section{Authors}

Simon D. Schnell - Department of Chemistry, University of Zurich, Winterthurerstrasse 190, 8057, Zurich.

Dr. Mauro Schilling - Department of Chemistry, University of Zurich, Winterthurerstrasse 190, 8057, Zurich.

Dr. Jan Sklyaruk - Department of Chemistry, University of Zurich, Winterthurerstrasse 190, 8057, Zurich.

Prof. Dr. Anthony Linden - Department of Chemistry, University of Zurich, Winterthurerstrasse 190, 8057, Zurich.

\section{Acknowledgements}

We would like to thank Joana Hauser for laboratory assistance and Dr. Jorge Gonzalez for insightful and thought-provoking discussions. 


\section{References}

[1] R. J. Ouellette, J. D. Rawn, in Principles of Organic Chemistry (Eds.: R. J. Ouellette, J. D. Rawn), Elsevier, Boston, 2015, pp. 133-162; A. J. Rocke, Angew. Chem. Int. Ed. 2015, 54, 46-50.

[2] a) R. V. Ottenbacher, E. P. Talsi, K. P. Bryliakov, Appl. Organomet. Chem. 2020, 34, e5900; b) W. A. L. van Otterlo, C. B. de Koning, Chem. Rev. 2009, 109, 3743-3782; c) T. L. Gilchrist, J. Chem. Soc., Perkin Trans. 1 2001, 2491-2515.

[3] K.-T. Chung, J. Environ. Sci. Health C 2016, 34, 233-261.

[4] a) M. D. Polêto, V. H. Rusu, B. I. Grisci, M. Dorn, R. D. Lins, H. Verli, Front. Pharmacol. 2018, 9, 395; b) R. D. Taylor, M. MacCoss, A. D. G. Lawson, J. Med. Chem. 2014, 57, 5845-5859; c) C. G. Wermuth, MedChemComm 2011, 2, 935-941; d) D. Dalvie, S. Nair, P. Kang, C.-M. Loi, in Metabolism, Pharmacokinetics and Toxicity of Functional Groups: Impact of Chemical Building Blocks on ADMET, The Royal Society of Chemistry, 2010, pp. 275-327; L. A. Thompson, J. A. Ellman, Chem. Rev. 1996, 96, 555-600.

[5] a) Modern Crop Protection Compounds, Third Edition, (Eds. W. Krämer, U. Schirmer, P. Jeschke, M. Witschel), Wiley-VCH, Weinheim, 2019; b) R. Beaudegnies, A. J. F. Edmunds, T. E. M. Fraser, R. G. Hall, T. R. Hawkes, G. Mitchell, J. Schaetzer, S. Wendeborn, J. Wibley, Bioorg. Med. Chem. 2009, 17, 4134-4152.

[6] a) J. McMurry, Organic Chemistry, Cengage Learning, Bosten, MA, 2016; b) J. D. Hepworth, M. J. Waring, D. R. Waring in Aromatic Chemistry (Ed. E. W. Abel), RSC, 2002; c) K. Hemming, The Chemical Educator 2001, 6, 396-398; d) R. C. Larock, Comprehensive Organic Transformations, Wiley-VCH, Weinheim, 1989.

[7] a) S. Rohrbach, A. J. Smith, J. H. Pang, D. L. Poole, T. Tuttle, S. Chiba, J. A. Murphy, Angew. Chem. Int. Ed. 2019, 58, 16368-16388; b) C. N. Neumann, J. M. Hooker, T. Ritter, Nature 2016, 534, 369-373; c) N. Chéron, L. El Kaïm, L. Grimaud, P. Fleurat-Lessard, Chem. Eur. J. 2011, 17, 14929-14934.

[8] J. F. Bunnett, R. E. Zahler, Chem. Rev. 1951, 49, 273-412.

[9] a) M. Baidya, F. Brotzel, H. Mayr, Org. Biomol. Chem. 2010, 8, 1929-1935; b) A. Sakakura, K. Kawajiri, T. Ohkubo, Y. Kosugi, K. Ishihara, J. Am. Chem. Soc. 2007, 129, 14775-14779; c) W. Steglich, G. Höfle, Angew. Chem. Int. Ed. 1969, 8, 981-981.

[10] A. Pinner, Ber. Dtsch. Chem. Ges. 1893, 26, 2126-2135.

[11] B. L. Oliveira, Z. Guo, G. J. L. Bernardes, Chem. Soc. Rev. 2017, 46, 4895-4950.

[12] J. Sauer, D. K. Heldmann, J. Hetzenegger, J. Krauthan, H. Sichert, J. Schuster, Eur. J. Org. Chem. 1998, 1998, 2885-2896. 
[13] L. Hong, S. Ahles, M. A. Strauss, C. Logemann, H. A. Wegner, Org. Chem. Front. 2017, 4, 871875.

[14] J. Faragó, Z. Novák, G. Schlosser, A. Csámpai, A. Kotschy, Tetrahedron 2004, 60, 1991-1996.

[15] A. R. Katritzky, S. Rachwal, R. J. Offerman, Z. Najzarek, A. K. Yagoub, Y. Zhang, Chem. Ber. 1990, 123, 1545-1552.

[16] D. Hunter, D. G. Neilson, J. Chem. Soc., Perkin Trans. 1 1984, 2779-2783.

[17] F. A. Neugebauer, R. Siegel, Chem. Ber. 1985, 118, 2157-2163.

[18] S.-E. Suh, S. Chen, K. N. Houk, D. M. Chenoweth, Chem. Sci. 2018, 9, 7688-7693.

[19] Y. Qu, P. Pander, O. Vybornyi, M. Vasylieva, R. Guillot, F. Miomandre, F. B. Dias, P. Skabara, P. Data, G. Clavier, P. Audebert, J. Org. Chem. 2020, 85, 3407-3416.

[20] a) S. D. Schnell, L. V. Hoff, A. Panchagnula, M. H. H. Wurzenberger, T. M. Klapötke, S. Sieber, A. Linden, K. Gademann, Chem. Sci. 2020, 11, 3042-3047; b) E. Ros, M. Bellido, X. Verdaguer, L. Ribas de Pouplana, A. Riera, Bioconjug. Chem. 2020, 31, 933-938; c) A. Counotte-Potman, H. C. Van der Plas, B. Van Veldhuizen, C. A. Landheer, J. Org. Chem. 1981, 46, 5102-5109.

[21] a) L. R. Domingo, P. Pérez, J. A. Sáez, RSC Adv. 2013, 3, 1486-1494; b) L. R. Domingo, M. J. Aurell, P. Pérez, R. Contreras, J. Phys. Chem. A 2002, 106, 6871-6875; c) Luis R. Domingo, Eur. J. Org. Chem. 2004, 2004, 4788-4793.

[22] P. Geerlings, F. De Proft, W. Langenaeker, Chem. Rev. 2003, 103, 1793-1874.

[23] D. Svatunek, C. Denk, H. Mikula, Monatsheft. Chem. 2018, 149, 833-837.

[24] a) P. Vermeeren, S. C. C. van der Lubbe, C. Fonseca Guerra, F. M. Bickelhaupt, T. A. Hamlin, Nat. Prot. 2020, 15, 649-667; b) I. Fernández, F. M. Bickelhaupt, Chem. Soc. Rev. 2014, 43, 4953-4967.

[25] D. S. Timofeeva, R. J. Mayer, P. Mayer, A. R. Ofial, H. Mayr, Chem. Eur. J. 2018, 24, 59015910.

[26] M. Balkenhohl, H. Jangra, T. Lenz, M. Ebeling, H. Zipse, K. Karaghiosoff, P. Knochel, Angew. Chem. Int. Ed. 2019, 58, 9244-9247.

[27] A. Hetzheim, D. Schneider, Zeitschrift für Chemie 1975, 15, 219-220.

[28] F. Corral-Bautista, L. Klier, P. Knochel, H. Mayr, Angew. Chem. Int. Ed. 2015, 54, 12497-12500. 